Sonderdruck aus

Christian Moser / Linda Simonis (Hg.)

\title{
Figuren des Globalen
}

Weltbezug und Welterzeugung in Literatur, Kunst und Medien

Mit 21 Abbildungen

V\&R unipress

ISBN 978-3-8471-0170-3

ISBN 978-3-8470-0170-6 (E-Book 



\section{Inhalt}

Christian Moser / Linda Simonis

Einleitung: Das globale Imaginäre . . . . . . . . . . . . . . . 11

Teil I: Figuren des Globalen, Narrative der Totalisierung

Christian Moser

Figuren des Globalen. Von der Weltkugel zum Welthorizont . . . . . . 25

Robert Stockhammer

Welt oder Erde? Zwei Figuren des Globalen . . . . . . . . . . . . . . . . . 47

Michael Auer

Präfigurationen des Planetarischen: Ernst Jünger, Gayatri Spivak und die typologische Lektüre . . . . . . . . . . . . . . . 73

Achim Hölter

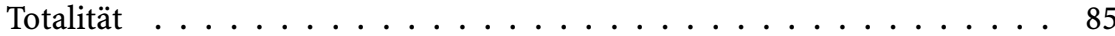

Kirsten Kramer

Globalität und Weltbezug in der französischen Kulturanthropologie und der spanischen Erzählliteratur der Gegenwart ． . . . . . . . . . . . . 105

Dominik Schreiber

Der Klimawandel - Aufstieg eines globalen Narrativs . . . . . . . . . . . 129

Teil II: Rekonzeptualisierungen von Weltliteratur

Erhard Schüttpelz

World Literature from the Perspective of longue durée . . . . . . . . . . . 141 
David Damrosch

Plus ça change? Die Komparatistik im globalen Zeitalter . . . . . . . . . 157

Dieter Lamping

Die Welt der Weltliteratur. Denotationen und Konnotationen eines suggestiven Begriffs . . . . . . . . . . . . . . . . . . . 169

Bernd Blaschke

Für? eine? Welt? -! Literatur? auf Französisch? Thesen und Fragen zum

Manifest von Michel Le Bris und seinen 43 . . . . . . . . . . . . . . . . 181

Joseph O’Neil

Nomos oder Medium der Erde? Zur Geopoetik der Weltliteratur . . . . . 193

\section{Teil III: Poetiken des Globalen}

David Damrosch

Geopoetics: World Literature in the Global Mediascape . . . . . . . . 209

Frederike Felcht

Eine globale Gegenwartshymne - Zur Poetologie von H. C. Andersens

Det nye Aarhundredes Musa [Die Muse des neuen Jahrhunderts] (1861) . 231

Ulrich Ernst

Eugen Gomringer und das Konzept einer Globalisierung der Poesie. Eine

Re-Lektüre des Manifests vom vers zur konstellation . . . . . . . . . . . 243

Beatrice Nickel

Avantgarde-Lyrik und Universalsprache: Die Konkrete Poesie in Brasilien und Frankreich als globales Phänomen . . . . . . . . . . . . 263

\section{Teil IV: ,Weltgenres‘}

Karl Maurer

Die Divina Commedia als Weltgedicht . . . . . . . . . . . . 281

Joachim Harst

Welttheater und Weltmacht. Christlicher Universalismus bei Gryphius und Calderón . . . . . . . . . . . . . . . . . . . . . . . . 289

Kristina Mendicino

Break-Dance. (Ein Schritt von Homer und Rousseau zu Goethe) . . . . . 301 
Alexander Nebrig

Die Welt als Lied. Der globale Anspruch von Herders Volksliedern . . . . 315

Martin Götze

Das Gedicht als ästhetische Rede. Zum Problem der Welthaltigkeit von Lyrik . . . . . . . . . . . . . . . . . . . . . . 327

Teil V: Fiktionen des Globalen: Zwischen Weltbezug und Welterzeugung

Christine Ivanovic

Weltgeschichte und Weltliteratur. Hannah Arendts „Welt“-Konzept im Kontext ihrer literarischen Analysen . . . . . . . . . . . . . 341

Barbara Ventarola

Zwischen situationaler Repräsentation und Multiadressierung - Marcel

Proust und Jorge Luis Borges als Paradigmen der Weltliterarizität . . . . 353

Alice Stašková

Zum Weltbezug als Textbezug des modernen Romans (Hermann Broch -

Georges Perec - Michal Ajvaz) . . . . . . . . . . . . . . . 369

Christian Sinn

Bilokationen. Literarische und mathematische Verfahren der

Welterzeugung in Thomas Pynchons Against the day . . . . . . . . . . 381

Evi Zemanek

Die generativen Vier Elemente: Zu einer Grundfigur der Welt- und

Text-Schöpfung am Beispiel von Franz Josef Czernins elemente-Sonetten 401

Christiane Solte-Gresser

Lebens-Welt-Verlust? Literarische Formen postmoderner Welterzeugung am Beispiel von Marlene Streeruwitz . . . . . . . . . . . . . . . . . 413

\section{Teil VI: Literarische Repräsentationen von Globalität und Globalisierung}

Dolf Oehler

Zur Dialektik der Globalisierung . . . . . . . . . . . . . . 427 
Nina Peter

„The Right Places at the Right Times“. David Mitchells Ghostwritten als Roman über die Denkbarkeit von Globalität . . . . . . . . . . . . . . . 439

Claudia Schmitt

Die Welt - ein Mosaik? Episodenhaftes Erzählen in Literatur und Film der Gegenwart . . . . . . . . . . . . . . . . . . . 455

Anne-Rose Meyer

Der Schriftsteller als Zeuge und Zuschauer. Die Beispiele Hans Christoph

Buch und Nick McDonell . . . . . . . . . . . . . . . . . . . . 467

\section{Teil VII: Globalität und (Inter-)Medialität}

Arndt Niebisch

Medienzusammenbrüche und posthumanes Erzählen in Jules Vernes

Michel Strogoff . . . . . . . . . . . . . . . . . . 483

Monika Schmitz-Emans

Welt-Bilder, Bildstile, Schreibstile. Hybridkulturelle Bildwelten und ihre literarische Beschreibung bei Orhan Pamuk . . . . . . . . . . . 495

Kirsten von Hagen

"Jeder ist überall, niemand irgendwo" - Weltwahrnehmung und

-konstruktion bei Daniel Kehlmann (Ruhm, 2009) und Giulio Minghini

(Fake, 2009) . . . . . . . . . . . . . . . 509

Frauke Bolln

Welt und Provinz in Text und Bild bei Dorothee Elmiger und Stefan

Ettlinger ............................ 523

Teil VIII: Geographie - Kartographie - Geopoetik

Angela Oster

Globalität und Globus. Technikfaszination und Kunsthandwerk der

Globographie in der Frühen Neuzeit . . . . . . . . . . . . . . 535

Dana Bönisch

Andere Karten. Videogeographie, Kartographie und Geopoetik . . . . . . 555 
Simon Harvey

Twisted Logics: A Topological Turn in Counter-Cartography and Some

Artistic Antecedents . . . . . . . . . . . . . . . . . 567

Teil IX: Weltwissen, Weltdiskurse, globale Zirkulation

Ulrike Kruse

Das Haus als Welt. Die geordnete Welt in der frühneuzeitlichen

Ökonomikliteratur . . . . . . . . . . . . . . . . . . . . 579

Andreas Beck

Welthandelswege im Märchenwald - Johann Carl August Musäus'

Stumme Liebe

Peter Goßens

,Eisenbahnen und Dampfschiffe‘. Zur Rolle der technischen

Fortbewegung im transnationalen Literaturdenken des frühen

19. Jahrhunderts . . . . . . . . . . . . . . . 603

Uwe Lindemann

Madame Bovary und der moderne Hedonismus. Reflexionen zum

Verhältnis von Literatur, globalisierter Warenwelt und Konsumkultur im

19. Jahrhundert . . . . . . . . . . . . . . . . . 615

Nicole Pöppel

Weltausstellungen als Schreibstätten des Globalen . . . . . . . . . . 633

Simone Sauer-Kretschmer

Die Inszenierung von Welt und ihre Grenzen - Ödön von Horváths

Alfons Kobler zu Besuch auf der Weltausstellung in Barcelona . . . . . . 645

Keyvan Sarkhosh

Die Welt als Archiv - Stanley Kubricks Napoleon-Projekt . . . . . . . . . 657

\section{Teil X: Verhandlungen kultureller Differenz im Spannungsfeld von Globalität und Lokalität}

Elke Brüggen

Belacâne, Feirefîz und die anderen. Zur Narrativierung von

Kulturkontakten im Parzival Wolframs von Eschenbach . . . . . . . . . 673 
Michael Bernsen

Gérard de Nervals Begegnung mit dem Orient, ein Globalisierungsschub in der französischen Literatur des 19. Jahrhunderts . . . . . . . . . 693

Marc Maufort

Forging Native Idioms: Canadian and Australasian Performances of Indigeneity in an Age of Globalization $\ldots \ldots \ldots \ldots 70 \ldots$

Christian Luckscheiter

Topographien Peter Handkes zwischen Lokalität und Globalität . . . 717

Zu den Beiträgerinnen und Beiträgern . . . . . . . . . . . . . 729 


\section{Welttheater und Weltmacht. Christlicher Universalismus bei Gryphius und Calderón}

I.

In der ersten Abhandlung von Andreas Gryphius' Märtyrerspiel Catharina von Georgien $^{1}$ steht der persische Tyrann Abas vor einer unerhörten Situation: Obwohl er mächtiger als jeder andere weltliche Fürst ist, schlägt seine Gefangene, die christliche Königin Catharina, sein Werben um ihre Hand unbewegt aus. Der resultierende harte Gegensatz zwischen Heide und Christ führt schließlich zur Hinrichtung der Christin, die den Märtyrertod in Kauf nimmt, um Keuschheit und Glauben zu bewahren, und dementsprechend nach christlicher Logik über die Tyrannei des Fleisches zu triumphieren. Um dies auch dem Zuschauer augenfällig zu bestätigen, sucht sie Abas in der Schlussszene als Gespenst heim und sagt ihm ein kurzes, aber qualvolles Leben zur Strafe seiner Sünden voraus. So endet das Spiel mit der Apotheose der Gefangenen und der Verdammung des Tyrannen: Kaum könnte die universale Geltung der christlichen Religion deutlicher exemplifiziert werden.

Seine Begründung erhält dieser Geltungsanspruch bereits in der Stichomythie, die aus der ersten Konfrontation zwischen Heide und Christ resultiert. Die dabei angewendete theologisch-juristische Gedankenfigur zeigt zugleich die Grundform der Problematik christlichen Universalismus auf, die ich in diesem Beitrag diskutieren möchte. Catharina weist den Antrag des Tyrannen mit dem Hinweis ab, „der Christen Recht verknüpfft nur Zwey durch dieses [nämlich der Ehe] Band" (1,781), der Perserfürst aber sei bereits mit mehreren Frauen verheiratet und komme daher für eine christliche Ehe nicht mehr in Frage. Abas

1 Das Trauerspiel wurde 1647 verfasst, aber erst 1657 veröffentlicht. Ich zitierte nach der Ausgabe Gryphius 1966. Nachweise von Zitaten aus dieser Ausgabe erfolgen fortan parenthetisch im fortlaufenden Text unter Angabe des Aktes und des Verses. Die Verszählung beginnt in Powells Ausgabe mit jedem Akt neu. Im Folgenden stelle ich eine Detailskizze zu einem Grundproblem barocken Theaters vor, das ich umfassender in meinem Buch Heilstheater. Figur des barocken Trauerspiels zwischen Gryphius und Kleist (2012a) untersucht habe. Eine wichtige Gesamtinterpretation des Stücks liefert Schings 1968. 
stellt daraufhin die Gültigkeit des christlichen Rechts in Frage: „Der Persen Recht gilt mehr. Wir sind in ihrem Land!“ (1,782; Hervorhebung J. H.) Doch dieses Argument, das die Rechtsgültigkeit mit dem Territorialbesitz verbindet, wird von Catharina augenblicklich gegen den Heiden gewendet: „Noch mehr des Höchsten Recht! wir stehn auff seiner Erden.“ (1,783; Hervorhebung J. H.) Der kurze Schlagabtausch zeigt schön, wie im barocken Trauerspiel der stichomythische Konflikt als rein quantitatives Überbieten verstanden wird: So wird Gott in eine Linie mit dem ungläubigen Tyrannen gestellt und unterscheidet sich zunächst nur dadurch von ihm, dass sein Recht mehr Geltung hat, weil er eben die ganze Erde besitzt. In Konsequenz wäre dem christlichen Gott auch der heidnische Herrscher unterworfen; dessen Recht, soweit es dem christlichen widerspricht, wäre grundsätzlich und paradoxal ,ungerechtes Recht“ (1,681). So wird es von den Christen denn auch genannt.

In dem widersprüchlichen Begriff eines „ungerechten Rechts“ tritt die Problematik eines christlichen Universalismus, wie ihn das Märtyrerspiel veranschaulicht, deutlich zutage. Eine tatsächlich universelle Gültigkeit des christlichen Rechts kann nur dargestellt werden, wenn es nicht allein die Gläubigen, sondern eben auch die Ungläubigen umfasst; letztere haben ebenfalls, obschon sie es nicht wissen, dem christlichen Gott zu unterstehen. ${ }^{2}$ Da sie aber zugleich von den Gläubigen deutlich geschieden werden müssen, resultiert die umfassende Vereinnahmung in einer inneren Spaltung des zugrunde liegenden Rechtsbegriffs. Sie kommt bei Gryphius in dem Oxymoron eines heidnischen „ungerechten Rechts“ einerseits, dem Pleonasmus eines christlichen „rechten Rechts" andererseits deutlich zum Ausdruck. So ruft Abas, durch die Erscheinung von Catharinas Geist im Schlussakt unwillkürlich, wenn auch vergeblich bekehrt, reumütig aus: „Schauet! Schaut! Der Himmel bricht! / Die Wolckenfeste reist entzwey / Das rechte Recht steht ihrer Sachen bey!“ (5,385 - 387)

Im Folgenden möchte ich zeigen, inwiefern diese Selbstentzweiung des Rechts Voraussetzung der anschaulichen Darstellung von universalistischen Geltungsansprüchen ist. Dabei gehe ich von der Überlegung aus, dass die Entzweiung des Oxymorons und die Dopplung des Pleonasmus sich als Figuren der Theatralisierung verstehen lassen, wenn man letztere als Andeutung einer Unterscheidung zwischen Maske und Wesen, Schein und Sein versteht. Das „ungerechte Recht“ ist ganz offensichtlich nur scheinbar „Recht“ zu nennen, während das „rechte Recht“ aufgrund der pleonastischen Formulierung die Möglichkeit seines Gegenteils bekundet. Wenn aber auch das „ungerechte Recht“

2 Die Problematik des christlichen Universalismus, wie sie sich hier abzeichnet, lässt sich bis zu Paulus zurückverfolgen; dort ist die Argumentation jedoch bezeichnenderweise umgekehrt ausgerichtet: Der Messias hebt das mosaische Gesetz auf und bricht den Bund mit dem erwählten Volk, um auch die Heiden in ihn eintreten zu lassen. Vgl. bspw. Röm 11,25 f. und dazu Agamben 2000 und Badiou 1997. 
noch Recht heißen darf, lässt sich nicht endgültig zwischen Recht und Unrecht scheiden. Daher verschärft sich das Problem der Theatralität zu einer Unentscheidbarkeit zwischen Schein und Sein, für die nicht die Maske, sondern das Gespenst die angemessene Repräsentation wäre. Das Gespenst nämlich, wie es bei Gryphius auftritt, ist zugleich Schein und Sein: Die Wiederkehr von Catharinas rächendem Geist und ihre Verdammung des Tyrannen bewährt das Recht der Christin mit der absoluten Autorität des Jenseits; zugleich aber ist das Gespenst als Wahnerscheinung des von Gewissensbissen gepeinigten Tyrannen zu deuten. Apodiktische Objektivität und wahnhafte Subjektivität sind daher im Gespenst untrennbar verbunden.

Aus dieser Beobachtung möchte ich den vorläufigen Schluss ziehen, dass der Universalitätsanspruch der christlichen Religion bei Gryphius mit der Problematik einer Theatralisierung zusammenhängt, die sowohl begrifflich als auch anschaulich zu fassen ist, in beiden Fällen aber den Bereich des Gespenstischen berührt: Die christliche Heilsgewissheit (als deren Ausdruck man das Märtyrerspiel üblicherweise versteht ${ }^{3}$ ) scheint sich nicht anders denn in der Erscheinung des Zweideutigen veranschaulichen zu können.

\section{II.}

Die Weltherrschaft christlichen Rechts hängt also auch bei Gryphius mit dem Problem einer Theatralisierung von Welt, einer Art „Welttheater“ zusammen, wenn es sich auch deutlich von dessen geläufigem Begriff unterscheidet, für den das Werk Calderóns repräsentativ ist. ${ }^{4}$ Dessen Comedia El príncipe constante (1636), die historische Ereignisse im Licht der christlichen Heilsgeschichte inszeniert, ${ }^{5}$ führt die juridisch-theologische Gedankenfigur aus dem Gryphschen Trauerspiel zum selbstbewussten Paradox fort; das führt auch zu einer Thematisierung von Theatralität, die schließlich - dies ist der gewichtige Unterschied gegenüber Gryphius - explizit für die universelle Geltung der christlichen Religion einsteht.

Fernando, ein portugiesischer Infante, ist nach der Eroberung Ceutas (1415)

3 Vgl. etwa Wiese 1967, S. 14, der den Wesensunterschied zwischen Tragödie und Trauerspiel eben in der „Heilsgewißheit“ des Christentums begründet: „Damit muß sich die Tragödie notwendig in Mysterienspiel, Passionsdichtung und Märtyrerdrama wandeln, Schmerz und Tod werden in einer Umkehr der Vorzeichen die Voraussetzung für das himmlische Heil [...] Hier findet eine Erlösung vom Tragischen statt.“

4 Man denke nur an dessen bekanntes Spiel El gran teatro del mundo. Vgl. zum barocken „Welttheater" allgemein Alewyn 1985.

5 Für eine detaillierte Interpretation der Comedia im Vergleich mit den historischen Quellen Calderóns vgl. Küpper 1990, S. $305-382$. 
in muslimische Gefangenschaft geraten; der maurische König will ihn dazu einsetzen, die Übergabe der Stadt Ceuta zu erzwingen, doch Fernando schlägt die Befreiung aus: Um die gerade errichtete Kirche in Nordafrika nicht preiszugeben, will er Gefangenschaft und Tod in Kauf nehmen. Mit dieser Geste will er Gott in einem ganz juristischen Sinn verpflichten, ihm das ewige Leben zu schenken. ${ }^{6}$ Wenn daher Fernando in einer zentralen Passage den maurischen König um „piedad“, 7 also Milde und Barmherzigkeit bittet, so muss man mit einem Hintergedanken Fernandos rechnen: Der Infante kann gar nicht wollen, dass ihm der maurische König das Leben schenkt, denn damit hätte er die Chance auf das ewige Leben verspielt.

Was also kann „piedad“ im vorliegenden Kontext bedeuten? Fernando begründet seine Bitte unter Bezugnahme auf ein universales Naturgesetz („ley de naturaleza“), dem auch der maurische König unterworfen sei. Als Beispiel nennt Fernando den Löwen, König der Tiere: Selbst die Bestie sei dieser „ley de naturaleza" unterworfen, verschone großmütig den deutlich Unterlegenen und zeige darin die ihrem königlichen Status entsprechende „piedad. ${ }^{\text {(8 }}$ Als weitere Beispiele werden Delphin, Adler, ja sogar der Granatapfel und der Diamant genannt: Sie alle würden den ihnen zukommenden Adel durch „piedad“ beglaubigen. Woraus Fernando schließt:

Pues si entre fieras y peces,

plantas, piedras y aves usan

esta majestad de rey,

la piedad, no será injusta

entre los hombres, señor,

porque el ser no te disculpa

de otra ley, que la crueldad

en qualquiera ley es una. (vv. $2476-2483$ )

Belege für ein solches „Naturgesetz“ findet man wohl nur in barocken Emblembüchern; ${ }^{9}$ im vorliegenden Zusammenhang aber ist vor allem die Zweideutigkeit der zentralen Begriffe entscheidend, mit denen Fernando seine Argumentation verfolgt: „piedad“ bezeichnet ja nicht nur „Milde“ oder „Barmherzigkeit“, sondern auch „Frömmigkeit“ im religiösen Sinn $;^{10}$ und „ley“ wird

6 So äußert Fernando die Hoffnung, „que aunque hoy cautivo muero, / rescatado he de gozar / el sufragio del altar, / que, pues yo os he dado / a vos tantas Iglesias, mi Dios, / alguna me habéis de dar" (vv. 2660 - 2662).

7 Vv. 2395 - 2607. Vgl. dazu auch Küpper 1990, S. 370 - 373.

8 „y así vemos / en repúblicas incultas / al León rey de las fieras, / que cuando la frente arruga / de guedejas, se corona; / es piadoso, pues que nunca / hizo presa en él, rendido“ (vv. 2428 2434).

9 Vgl. dazu Brancaforte 1997.

10 Vgl. Diccionario de la lengua española 1992, s. v. „piedad“: „1. f. Virtud que inspira, por el 
im vorliegenden Kontext zugleich als „(Natur-)Gesetz“ und als „Glauben“ verwendet. ${ }^{11}$ So erscheint die Milde des Löwen zugleich als verborgene Frömmigkeit - das „Naturrecht“, für das er steht, ist religiös grundiert. Daher kann der Maure als „de otra ley“ anerkannt werden, wird aber nichtsdestotrotz über die „ley de naturaleza" unter der Hand christianisiert: Von ihm wird dieselbe (christliche) Frömmigkeit erwartet, die auch dem Löwen eignet. Ähnlich wie der Tyrann Abas kann sich der Maurenkönig dem christlichen Anspruch nicht entziehen.

Damit ist die Zweideutigkeit des Begriffes „piedad“ aber noch nicht ausgeschöpft. Nachdem Fernando die Verpflichtung des Königs zur Milde aus dem Naturrecht hergeleitet hat, vollzieht er eine abrupte Kehrtwendung: Nicht um das Leben, sondern um den Tod bitte er. Selbst wenn der König sich also der natürlichen „piedad“ widersetzte und den Infanten im Jähzorn hinrichten ließe, hätte er in bestialischer „crueldad“ nur den himmlischen Willen und Fernandos „deseo de morir / por la Fe“ (vv. 2533 f.) erfüllt. Weil der Märtyrertod aber als christliches Opfer („sacrificio“) verstanden wird, würde die Grausamkeit des Königs nur der Verherrlichung Gottes und mithin der „piedad“ dienen: „No has de triunfar de la Iglesia, / de mí, si quisieres, triunfa; / Dios defenderá mi causa, / pues yo defiendo la suya" (vv. 2574 - 2577). Damit sind dem Heiden sämtliche „heidnischen“ Handlungsoptionen genommen: Egal wie er sich verhält, seine Reaktion wird der Verherrlichung des christlichen Gottes dienen. ${ }^{12}$

Diese an einer konkreten Textstelle entwickelte universalisierende Strategie der Comedia kann auch allgemeine Geltung für Calderóns Geschichtsverständnis und besonders seinen Umgang mit dem Phänomen beginnender Globalisierung beanspruchen: Die mit der europäischen Expansion entdeckten ,neuen Welten', deren schiere Existenz den christlichen ,ordo'-Gedanken erschüttern musste, insofern sie eine potentiell unendliche Pluralisierung der Welt

amor a Dios, tierna devoción a las cosas santas, y, por el amor al prójimo, actos de amor y compasión. 2. f. Amor entrañable que consagramos a los padres y a objetos venerandos. 3. f. Lástima, misericordia, conmiseración."

11 Vgl. ebd., s. v. „ley“: „1. f. Regla y norma constante e invariable de las cosas, nacida de la causa primera o de las cualidades y condiciones de las mismas. [...] 4. f. En el régimen constitucional, disposición votada por las Cortes y sancionada por el jefe del Estado. 5. f. Religión, culto a la Divinidad.“

12 Dass die Heiden bei Calderón grundsätzlich in das christliche Weltbild einbezogen sind (und nicht wie bei Gryphius außerhalb seiner stehen), zeigt sich auch an der Figurenkonstellation: Der Christusfigur Fernando steht die maurische Prinzessin gegenüber, deren Name Fénix auf eine traditionelle Christusallegorie verweist - der Vogel Phoenix, der aus seiner Asche neu geboren wird, veranschaulicht die Auferstehung Christi (vgl. bspw. Physiologus, S. 14 - 17). Darin zeigt sich die heidnische Welt als der christlichen grundsätzlich kompatibel; in ihr wird nicht (wie bei Gryphius) das widerchristliche Böse, sondern der noch unerlöste Mensch und mithin eine Phase der Heilsgeschichte veranschaulicht, die durch Fernando / Christus überwunden werden wird. 
nahelegen. ${ }^{13}$ Sie kann nur durch ein Geschichtsbild gesteuert werden, das im ,Neuen' den ,Schatten' des Alten erkennt und es auf diese Weise in die vorausgesetzte Ordnung einbettet. Aus Calderóns

Sicht sind die Neue Welt und die dort sich ereignende Geschichte eben nicht jenes ,aliquid de novo', dessen nunmehr erwiesene Existenz der nominalistischen Prämisse zu definitivem Triumph verhelfen könnte, sie sind vielmehr nichts anderes als eine ,Ausprägung' der für alle Zeit geordneten und insofern bereits gewußten Welt. Calderón beläßt den Neuen Welten den Reiz des Neuen und des Individuellen, aber er enthüllt ihn zugleich als ,umbra', durch die der stets identische Typos hindurchscheint. ${ }^{14}$

Der christliche Universalismus geht bei Calderón also deutlicher als bei Gryphius nicht nur mit einem totalen religiösen Geltungsanspruch, sondern auch mit einer imperialistischen Globalisierungsbewegung zusammen: Auf seiner Grundlage ist eine Entdeckung (und Unterwerfung) des ,Neuen' denkbar, ohne zugleich die alte Ordnung zu schwächen.

Ähnlich wie bei Gryphius bleibt dagegen der Universalismus in der inneren Spaltung eines Begriffs begründet: Der Heide mag glauben, mit der Tötung des Christen eine grausame, unmenschliche und unfromme Tat zu begehen; der Märtyrer hingegen weiß sie als höchste Gnade und Frömmigkeit zu schätzen. „Crueldad“ wäre hier also der innere Gegensatz universaler „piedad“, der die Darstellung der letzteren erst ermöglicht: Kein Märtyrer könnte seinen Glauben auf der Bühne bewähren, gäbe es nicht den Tyrannen, dem er die Hinrichtung verdankt. Insofern Fernando dieses Abhängigkeitsverhältnis explizit macht, ist seine Rede an den maurischen Fürsten zugleich Reflexion auf die Grundstruktur des Märtyrerspiels; so bringt er gewissermaßen die Situation Catharinas auf den (freilich zweideutigen) Begriff.

Es ist daher nicht verwunderlich, dass auch Calderóns Comedia mit der Erscheinung eines Gespenstes endet. Wie Catharina bei Gryphius in der Schlussszene dem Tyrannen erscheint, um die absolute Gültigkeit des christlichen Rechts an dem Ungläubigen zu exemplifizieren, so kehrt auch der verstorbene Fernando christusgleich auf den irdischen Schauplatz zurück, um seine Glaubensbrüder und damit auch die Kirche zum Sieg gegen die Heiden zu führen. Der Zweideutigkeit des universalen Begriffes entspricht also erneut die Er-

13 Küpper verbindet daher die europäische Expansionsbewegung mit dem Gedanken Giordano Brunos, einem wahrhaft allmächtigen Gott müsse es freistehen, unendlich viele Welten $\mathrm{zu}$ schaffen. Die Konsequenz dieser Allmacht aber wäre „die Eskamotierung des personalen Gotteskonzepts“: „Ist die Erde nicht länger Zentrum eines wohlgeordneten, sondern kontingenter Bestandteil eines beliebigen Kosmos, wird die Annahme heilsgeschichtlicher Geordnetheit zu einer Anmaßung“ (Küpper 1990, S. 308).

14 Küpper 1990, S. 310. ,Umbra‘ und ,Typos' sind Begriffe aus der christlichen Figuraldeutung, vgl. dazu grundlegend Auerbach 1967. 
scheinung eines Gespenstes. Allerdings ist sie hier - anders als bei Gryphius nicht als subjektiver Wahn gefasst: Fernando erscheint nicht dem ungläubigen König allein, sondern auch den Christen; die Gültigkeit der christlichen „ley“ wird also nicht in einer Vision, sondern in einem objektiven Wunder demonstriert. Damit wird die Problematik der Theatralität jedoch nur scheinbar zurückgenommen: Da es sich bei dieser Stelle um eine offenkundige Modifikation der historischen Quellen seitens Calderóns handelt, ${ }^{15}$ wird hier eher Geschichte zur Darstellung des christlichen Heilsgeschehens theatralisiert: Die fundamentale Zweideutigkeit von „piedad“ kehrt in der Zweideutigkeit christlich verstandener Geschichte, in der eine brutale Niederlage als Triumph dargestellt werden kann, wieder. Auch aus diesem Grund kann man in Calderóns Welttheater eine Reflexion auf das Problem der Theatralität erkennen, wie ich es anfangs bei Gryphius vorgestellt habe.

\section{III.}

Um den Status der Theatralität bei Gryphius und Calderón noch genauer zu fassen, möchte ich abschließend einen kontrastierenden Vergleich mit einer antiken Tragödie ziehen, in der ebenfalls die Frage eines universalen Gesetzes auf dem Spiel steht: In der sophokleischen Antigone vertritt Kreon die Position, dass der weltliche Machthaber über die Bestattung bzw. Nicht-Bestattung der Kriegsleichen bestimmen kann, während Antigone für die universale Geltung des Bestattungsritus eintritt. Deren Darstellung setzt auch hier eine innere Spaltung voraus: Nur weil Polyneikes zum Verräter und Ausgestoßenen wurde, lässt sich jetzt der Anspruch stellen, dass ihm dennoch ein Begräbnis zuteil werde. Allerdings trägt sich diese Spaltung anders in die Personenkonstellation ein, als das beim barocken Trauerspiel der Fall gewesen ist: Während sich bei Gryphius Tyrann und Märtyrer antithetisch gegenüber stehen, ist der Verräter in Antigone Familienmitglied; zwar versucht Kreon, ihn durch die Verweigerung der Bestattung nachträglich aus der Familie auszuschließen, räumt aber in seiner eigenen Darstellung des Geschehens implizit ein, dass sich der Konflikt nicht antithetisch schematisieren lässt:

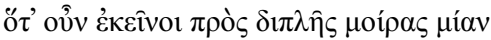

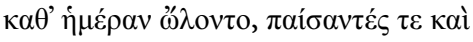

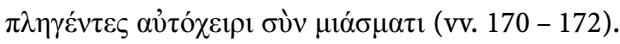

15 Bei Calderón muss sich der maurische König den portugiesischen Truppen unter der Führung Fernandos ergeben; historisch stehen der Tod Fernandos in der Gefangenschaft und der erste, zudem erfolglose Angriff auf Tanger (1437) in keinem Zusammenhang. Erst 1471 gelingt die Eroberung. 
Kreon betont in seiner Schilderung die Wechselseitigkeit der Tat: „schlagend und geschlagen in der eigenhändigen Befleckung“ seien die Brüder „durch doppeltes Schicksal an einem Tag zugrunde gegangen“; der Akzent liegt also auf der Feststellung, dass jeder der Brüder zugleich Handelnder und Leidender ist, ja dass hier das eigene Schlagen zugleich ein Geschlagen-Werden ist, weil es sich gegen den Bruder als ein anderes Selbst richtet. Aus diesem Grund lässt sich der Konflikt nicht in einem fremden Außerhalb lokalisieren, wie es der persische Tyrann bei Gryphius repräsentiert, sondern entzündet sich im inneren ,Selbst ${ }^{\star}$ der Tragödie - auch deshalb wird er als ",eigenhändige Befleckung“ bezeichnet. ${ }^{16}$

Sprachlich stellt sich diese innerliche Entzweiung in der Zweideutigkeit des zentralen Verbs $\sigma \varepsilon ́ \beta \varepsilon \imath v$ dar, das - ähnlich wie „piedad“ - Ehrfurcht vor Satzungen und Bräuchen ausdrückt. ${ }^{17}$ Der dem Wort implizite Gegensatz wird in der Stichomythie zwischen Kreon und Haimon ausgesprochen, wenn Kreon seine eigene Unnachgiebigkeit als ein Respektieren der politischen Herrschaft ( $\tau \dot{\alpha} \varsigma$ $\grave{\varepsilon} \mu \grave{\alpha} \varsigma \alpha \hat{\alpha} \chi \grave{\alpha} \varsigma \sigma \varepsilon \dot{\varepsilon} \beta \omega v)$ darstellt, der Sohn dagegen das Wort allein aus der religiösen Sphäre bestimmt:

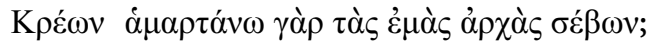

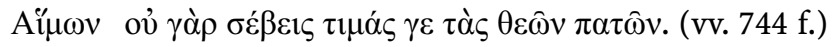

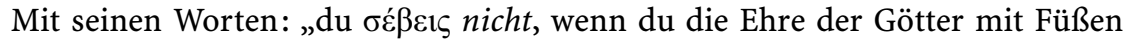
trittst“ stellt Haimon explizit die „Ehrfurcht“ des Vaters in Frage; der Konflikt

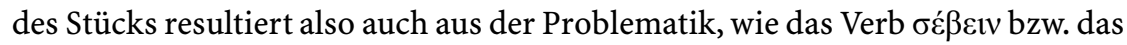

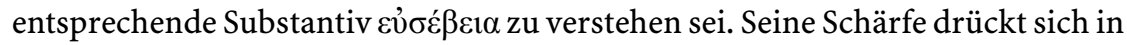
der verzweifelten Klage der verurteilten Antigone aus, ihr sei die Pflichterfüllung

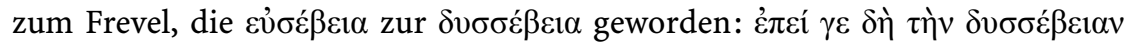

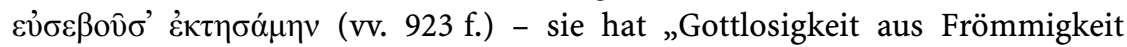
empfangen“, wie Hölderlin übersetzt. ${ }^{18}$

Freilich scheint das Ende der Tragödie mit dem Untergang Kreons Antigone

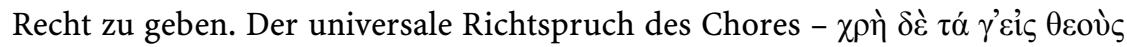

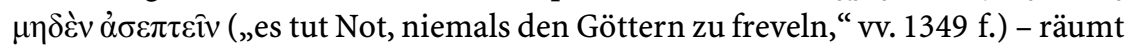
der religiösen Pflichterfüllung den Vorrang gegenüber der politischen ein, auch wenn der Chor auf der Hälfte des Stücks das genaue Gegenteil behauptet hat. Umso interessanter ist es, dass der Antagonismus zwischen Kreons und Antigones Deutung von $\varepsilon v ่ \sigma \varepsilon ́ \beta \varepsilon ı$ von einem Parallelismus ihrer Schicksale kom-

$16 \mathrm{Zu}$ dieser Auffassung des, Selbst' der Tragödie, untersucht am Beispiel des bemerkenswert

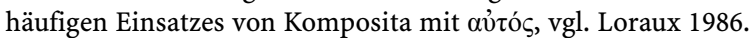

17 Das entsprechende Substantiv $\varepsilon \dot{\sigma} \sigma \varepsilon \varepsilon \varepsilon 1 \alpha$ wird folgendermaßen bestimmt: „1. reverence towards the gods or parents, piety or filial respect; 2. loyalty; 3. = lat. pietas“ (A Greek-English

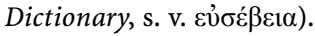

18 Hölderlin 1988, S. 361. 
plementiert wird, der sich in der Doppelstruktur der Tragödie ausdrückt: Während Antigone die erste Hälfte des Stücks bis zu ihrer Verurteilung dominiert, tritt Kreon in der zweiten Hälfte in den Vordergrund; weiterhin schließen beide ihre Bühnenexistenz mit Klagegesängen, in denen sie sich als lebende Tote darstellen. Das liegt in Bezug auf Antigone nahe, die ja zum Begräbnis bei lebendigem Leib verurteilt wurde, doch auch Kreon beschreibt sich zum Schluss als „zerstörten Mann“, der durch die Nachricht vom Selbstmord seiner Frau

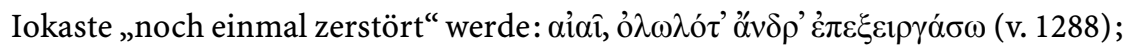
zuvor wurde er bereits von dem Boten als „beseelte Leiche“19 angesprochen. Sprachlich werden also beide Figuren in dem gespenstigen Bereich zwischen Lebenden und Toten angesiedelt; das verstehe ich als einen Hinweis darauf, dass sowohl die Protagonistin wie auch ihr Antagonist im Anspruch jenes univer-

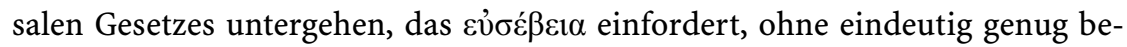
stimmt zu sein, um erfüllt werden zu können.

Zugleich hebt der Schluss der Tragödie noch einmal hervor, dass die Personenkonstellation aufgrund der Zweideutigkeit des Streitpunkts nicht antithetisch gedacht werden kann, sondern aus dem „Selbst“ der Tragödie entspringt: Kreon, der die Leiche seines Sohns in den Armen hält, beklagt sein Schicksal mit Worten, die seiner Beschreibung des Bruderkampfes strukturell analog sind: $\hat{\omega}$

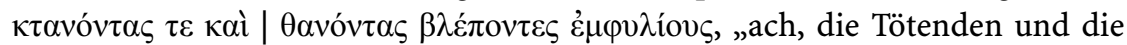
Getöteten seht ihr, gleichen Bluts“ (v. 1263 f.). Denn auch hier ist das Töten zugleich Getötet-Werden - sowohl im Selbstmord des Sohns ${ }^{20}$ wie auch in der Selbstwahrnehmung des Vaters, der sich als den Tötenden beschreibt und eben deswegen als Getöteten empfindet. Der Bruderkrieg, den Kreon durch sein Gesetz entscheiden wollte, setzt sich mithin strukturell im Schicksal des Königs fort; sein Versuch, eine endgültige Unterscheidung zwischen Recht und Unrecht durch den Ausschluss Polyneikes' einzuführen, wird durch die wiederholte problematische Identität von Täter und Opfer unterlaufen.

In dieser Perspektive würde also das sprachlich evozierte ,Gespenst ' in Antigone das genaue Gegenteil dessen bewirken, was es im christlichen Trauerspiel veranschaulichen soll: Wenn es dort dazu dient, den Triumph des christlich-

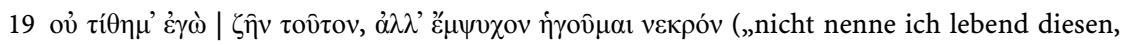
sondern halte ihn für einen beseelten Leichnam“, vv. 1166 f.).

20 Bezeichnenderweise fragt der Chor, nachdem der Tod Haimons ausdrücklich als „von ei-

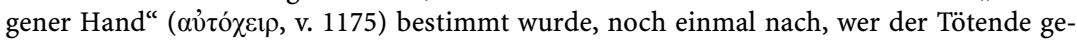
wesen sei - Sohn oder Vater; in beiden Fällen, so muss man schließen, läge jene Identität von Tötendem und Getötetem vor, die den Begriff Selbstmord rechtfertigte (v. 1175 f.; vgl. dazu Loraux 1986, S. 176 f.). Dass auch der Tod der Brüder Polyneikes und Eteokles als $\alpha$ vó $\chi \varepsilon ı 1 \rho$ bezeichnet wird, knüpft den Parallelismus noch enger (v. 172) und verbindet beide Stellen

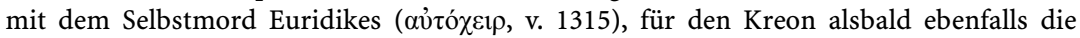

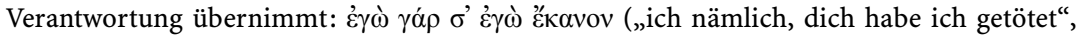
v. 1319). 
universalen Rechts und die Erlösung seiner Subjekte zu beglaubigen, so wird es in Antigone beschworen, um den Untergang der Figuren im Anspruch des universalen Gesetzes sprachlich vor Augen zu stellen; während ein zentraler Begriff der Tragödie trotz des Chorspruches zweideutig bleibt, zielt das Trauerspiel auf anschauliche Vereindeutigung. Aus diesem Grund nimmt man oft einen Wesensunterschied zwischen Tragödie und Trauerspiel an, der sich auch darin zeige, dass die christliche Heilsgewissheit die Zweideutigkeit des „tragischen Schicksals“ überwunden und damit, wie Benno von Wiese schreibt, eine „Erlösung vom Tragischen“ erreicht habe. ${ }^{21}$

Während dieser Gedanke auf theologischem Gebiet einsichtig sein mag, wird ihm auf der Bühne sowohl von der Tragödie als auch vom Märtyrerspiel durch das Gespenstische widersprochen. Denn der Konflikt in Antigone besteht ja gerade nicht in einer äußeren Schicksalhaftigkeit - die Protagonistin wird im

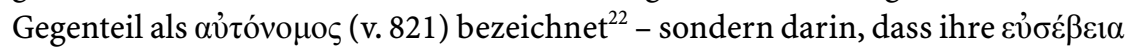
zugleich $\delta v \sigma \sigma \varepsilon ́ \beta \varepsilon ı \alpha$ ist. Antigone und Kreon werden „Gespenster“, weil die basale Unterscheidung zwischen Einhaltung und Bruch des universalen Gesetzes nicht aufrecht zu erhalten ist. Eine ähnliche Zweideutigkeit ist bei Calderón und Gryphius mit den Begriffen „piedad“ und „Recht“ zentral; doch hier soll die Zweideutigkeit - man möchte sagen: ausgerechnet - durch die Erscheinung des Gespenstes gelöst werden. Gerade das Gespenst als Manifestation des Zweideutigen kann aber die Ambivalenz nicht lösen, sondern höchstens gewaltsam ausschließen: Zwar regiert sie nicht mehr das „Selbst“ der Tragödie, aber wird nurmehr in den theatralen Apparat - $\mu \eta \chi \alpha v \eta ́$ und ő $\psi 1 \varsigma$ - des Trauerspiels verlagert. ${ }^{23}$ Hier soll der institutionierte Schein des Gespenstes für die umfassende Eindeutigkeit von „Recht“ und „piedad“ einstehen - was im Rückschluss bedeutet, dass die von solcher Frömmigkeit regierte Welt gespenstiges „Welttheater" wird. Die Affirmation dieses Zusammenhangs, die durch seine Umkehrung zum Gottesbeweis möglich wird (das Welttheater fordert einen Gott als Regisseur), macht den Erfolg der jesuitischen Mission aus: So bestätigt die Entdeckung und Unterwerfung jeder „Neuen Welt“ erneut den Universalismus des Christentums. Dessen tiefe Problematik wird besonders scharf bei Gryphius deutlich, der nicht ohne Grund jeden expliziten Hinweis auf das „Welttheater“ unterdrückt: Das Gespenst Catharinas, das Abbas verflucht und darin zugleich

21 Vgl. Wiese 1967, S. 14.

22 Wobei, wie deutlich geworden sein sollte, dies nicht als „Autonomie“ im modernen Sinn, sondern als Gesetz des (tragischen) Selbsts verstanden werden muss. Vgl. zur Vokabel auch Loraux 1986, S. $171 \mathrm{f}$.

23 In diesem (aristotelischen) Sinn kann man das Trauerspiel gegenüber der Tragödie als dasjenige Theater bestimmen, in dem die materiellen Rahmenbedingungen des Schauspiels einen zentralen, eben „erlösenden“ Stellenwert erhalten. Vgl. dazu ausführlich Harst 2012b und ferner Menke / Menke 2007, bes. S. 6-15. 
die Erlösung der Christin bewährt, ist als Wahnbild des Tyrannen beschrieben. Das christliche Publikum kann sich also der ihm verheißenen „Erlösung“ visuell nur versichern, wenn es den Standpunkt des Verdammten einnimmt.

\section{Literaturverzeichnis}

Agamben, Giorgio: Il tempo che resta. Un commento alla lettera ai Romani. Turin 2000. A Greek-English Dictionary. Hg. v. Henry G. Liddell u. Robert Scott. 9., von Henry S. Jones überarbeitete Aufl. Oxford 1973.

Alewyn, Richard: Das große Welttheater. Die Epoche der höfischen Feste. 2., erw. Aufl. München 1985.

Auerbach, Erich: „Figura“, in: Auerbach, Erich: Gesammelte Aufsätze zur romanischen Philologie. Hg. v. Fritz Schalk. Bern / München 1967, S. 55 - 93.

Badiou, Alain: Saint Paul, la fondation de l'universalisme. Paris 1997.

BranCAForte, Elio: „The Emblematic Tradition in Calderón's El Príncipe constante“, in: Ibero-Romania 1997/46, S. 40 - 59.

Calderón de la Barca, Pedro: El príncipe constante. Edición de Fernando Cantalapiedra y Alfredo Rodríguez López-Vázquez. Madrid 1996 [1636].

Ders.: El gran teatro del mundo. Edición, prólogo y notas de John J. Allen y Domingo Ynduráin. Con un estudio preliminar de Domingo Ynduráin. Barcelona 1997 [1655].

Diccionario de la lengua española. Hg. v. der Real academía española. Madrid ${ }^{21} 1992$.

Gryphius, Andreas: Catharina von Georgien [1657], in: Gryphius, Andreas: Gesamtausgabe der deutschsprachigen Werke. Hg. v. Marian Szyrocki u. Hugh Powell. Bd. 6: Trauerspiele 3. Hg. v. Hugh Powell. Tübingen 1966, S. $131-224$.

HARsT, Joachim: Heilstheater. Figur des barocken Trauerspiels zwischen Gryphius und Kleist. München 2012a.

Ders.: „Deus ex machina. Überlegungen zum Gott des barocken Trauerspiels am Beispiel von Gryphius, Heinsius und Racine“, in: Poetica 44/2012b, S. 351 - 378.

Hölderlin, Friedrich: Antigonä, in: Hölderlin, Friedrich: Sämtliche Werke. Historischkritische Ausgabe. Frankfurter Ausgabe. Hg. v. D. E. Sattler. Bd. 16: Sophokles. Frankfurt a. M. 1988, S. $264-407$.

KüPPER, Joachim: Diskurs-Renovatio bei Lope de Vega und Calderón. Untersuchungen zum spanischen Barockdrama. Mit einer Skizze zur Evolution der Diskurse in Mittelalter, Renaissance und Manierismus. Tübingen 1990.

Loraux, Nicole: „La main d'Antigone“, in: Metis 1986/1, S. 165 - 196.

Menke, Bettine / Menke, Christoph (Hg.): Tragödie, Trauerspiel, Spektakel. Berlin 2007. Physiologus. Griechisch/deutsch. Übers. u. hg. v. Otto Schönberger. Stuttgart 2001.

Schings, Hans-Jürgen: „Catharina von Georgien. Oder Bewehrete Beständigkeit“, in: Kaiser, Gerhard (Hg.): Die Dramen des Andreas Gryphius. Eine Sammlung von Einzelinterpretationen. Stuttgart 1968, S. 35 - 74.

Sophocles: Antigone. Ed. by Mark Griffith. Cambridge 1999.

Wiese, Benno von: Die deutsche Tragödie von Lessing bis Hebbel. Hamburg ${ }^{7} 1967$. 
